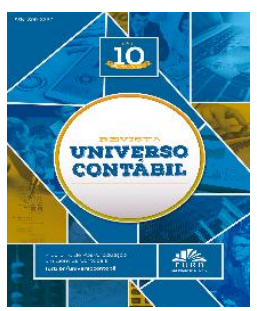

Revista Universo Contábil, ISSN 1809-3337

Blumenau, v. 12, n. 2, p. 21-38, abr./jun., 2016

doi:10.4270/ruc.2016212

Disponível em www.furb.br/universocontabil

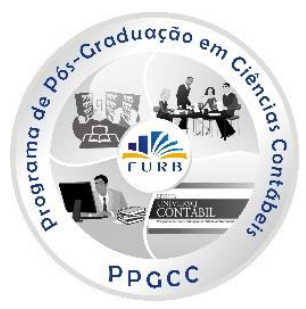

\title{
PERFIL DA GESTÃO DE CONSÓRCIOS INTERMUNICIPAIS DE SAÚDE DO RIO GRANDE DO SUL: UM ESTUDO DAS PRÁTICAS DE GESTÃO ECONÔMICA E FINANCEIRA ${ }^{1}$
}

\section{PROFILE OF MANAGEMENT INTERCITY HEALTH CONSORTIA OF RIO GRANDE DO SUL: A STUDY OF THE ECONOMIC AND FINANCIAL MANAGEMENT PRACTICES \\ PERFIL DE GESTIÓN DE CONSORCIOS INTERMUNICIPALES DE SALUD DEL RIO GRANDE DO SUL: UN ESTUDIO DE LAS PRÁCTICAS DE GESTIÓN ECONÓMICA Y FINANCIERA}

\author{
Hermes Cardoso Reis \\ Mestre em Ciências Contábeis pela Universidade do Vale do Rio dos Sinos - UNISINOS \\ Professor Assistente da Anhanguera Educacional \\ Endereço: Av. Cavalhada 4890, bairro Cavalhada \\ CEP 91.740-000 - Porto Alegre - RS - Brasil \\ E-mail: hermes.reis@anhanguera.com \\ Telefone: +55 (51) 3092-5700
}

Marcos Antônio de Souza

Doutor em Controladoria e Contabilidade pela Universidade de São Paulo - FEA/USP Professor e Coordenador do Programa de Pós-Graduação em Ciências Contábeis da Universidade do Vale do Rio dos Sinos - UNISINOS

Endereço: Av. Unisinos, 950, Cristo Rei CEP 93.022-000 - São Leopoldo - RS - Brasil

E-mail: marcosas@unisinos.br Telefone: +55 (51) 3037-1000 Ramal: 1580

Lauro Brito de Almeida Doutor em Controladoria e Contabilidade pela Universidade de São Paulo - FEA/USP Professor do Programa de Pós-Graduação em Contabilidade da Universidade Federal do Paraná (PPGCONT- UFPR)

Endereço: Av. Prefeito Lothário Meissner, 632, 1ำ andar, Sala 18, Campus III, Jardim Botânico CEP 80210-170 - Curitiba - PR - Brasil E-mail: gbrito@uol.com.br Telefone: +55 (41) 3360-4404

Débora Gomes Machado Doutora em Ciências Contábeis e Administração pelo Programa de Pós-Graduação em Ciências Contábeis (PPGCC) da Universidade Regional de Blumenau - FURB Professora do Programa de Pós-Graduação em Administração da Universidade Federal do Rio Grande - FURG

\footnotetext{
${ }^{1}$ Artigo recebido em 16.12.2015. Revisado por pares em 29.05.2016. Reformulado em 24.07.2016. Recomendado para publicação em 27.07.2016 por Paulo Roberto da Cunha. Publicado em 27.08.2016. Organização responsável pelo periódico: FURB.
} 
Endereço: Av Itália, KM 8, s/ nº Pav. 4, Campus Carreiros CEP 96.203-000 - Rio Grande - RS - Brasil E-mail: debora_furg@yahoo.com.br Telefone: +55 (53) 3293-5096

\section{RESUMO}

O objetivo deste estudo é analisar a utilização de práticas de gestão econômica e financeira por Consórcios Intermunicipais de Saúde (CIS), estabelecidos no Rio Grande do Sul. Os CIS são estruturas de cooperação regional e intergovernamental formados entre municípios para atuar de forma conjunta na área da saúde pública. As práticas de gestão econômica e financeira são vistas como importantes para apoiar o processo decisório visando ao planejamento, execução, controle e avaliação de desempenho das organizações em geral. Trata-se de um estudo qualiquantitativo, de natureza aplicada e descritiva, no qual as percepções dos gestores dos CIS quanto à utilização destas práticas de gestão foram coletadas por meio de instrumento de pesquisa formatado com escalas do tipo Likert e tratados de forma objetiva, com uso de técnicas de estatística descritiva e exploratória. Assim, os CIS foram classificados e agrupados conforme o grau de utilização das práticas de gestão. Os principais achados indicam que o CIS é uma instância de execução e realização e não, propriamente, de formular estratégias para reduzir custos e ampliar e qualificar a oferta do serviço público de atendimento à saúde. Por esta razão, faz uso reduzido das práticas de gestão econômica e financeira. Além disso, a preocupação precípua da gestão dos CIS é voltada para o cumprimento estrito das obrigações legais e normativas para si e para os municípios associados.

Palavras-chave: Gestão Econômica e Financeira. Consórcios Públicos. Gestão Pública.

\section{ABSTRACT}

The objective of this study is to analyze the use of economic and financial management practices by the Consórcios Intermunicipais de Saúde (CIS), established in Rio Grande do Sul. The CIS are regional and intergovernmental cooperation structures formed between municipalities to act jointly in the area of public health. The economic and financial management practices are seen as important elements to support the decision-making process aimed at planning, execution, control and evaluation of organizational performance in general. It is a qualitative and quantitative study of applied nature and descriptive, in which the perceptions of managers of the CIS regarding the use of these management practices were collected through research tools formatted with Likert scales and treated objectively, using descriptive and exploratory statistical techniques. Thus, the CIS were classified and grouped according to the intensity of management practices being used by these organizations. The main findings indicate that the CIS is an instance of execution and achievement and are not intended to be a place for formulating strategies to reduce costs and increase the quality of health care services being offered to public. For this reason, it makes little use of economic and financial management practices. In addition, the major concern of CIS management is to focused on strict compliance with legal and regulatory obligations to themselves and to the associated municipalities.

Keywords: Economic and Financial Management. Public Consortium. Public Administration.

\section{RESUMEN}

El objetivo de este estudio es analizar el uso de prácticas de gestión económica y financiera de los Consorcios Intermunicipales de Salude (CIS), establecida en Rio Grande do Sul, Brasil. El CIS son estructuras de cooperación regionales e intergubernamentales que se forman entre los ayuntamientos para actuar conjuntamente en el ámbito de la salud pública. Las prácticas de 
gestión económica y financiera se consideran importantes para apoyar el proceso de la toma de decisiones enderezadas a la planificación, ejecución, control y evaluación del desempeño de las organizaciones en general. Se trata de un estudio cualitativo y cuantitativo de carácter aplicado y descriptivo, en el que se recogieron las percepciones de los directivos de los CIS sobre el uso de estas prácticas de gestión a través de la herramienta de investigación formateado con escalas Likert y tratados de manera objetiva, utilizando técnicas estadísticas descriptivas y exploratorias. De este modo, el CIS fueron clasificados y agrupados de acuerdo con el grado de uso de las prácticas de gestión. Los principales resultados indican que el CIS es una instancia de ejecución y actuación y no, propiamente, para formular estrategias para reducir los costos y ampliar y cualificar la prestación del servicio público de atención médica. Por esta razón, no tiene mucho uso de prácticas de gestión económica y financiera. Además, la preocupación de la gestión de la CIS se centra en el cumplimiento estricto de las obligaciones legales y reglamentarias a sí mismos y a los municipios asociados.

Palabras clave: Gestión Económica y Financiera. Consorcios Públicos. Gestión Pública.

\section{INTRODUÇÃO}

A Constituição brasileira de 1988 prevê a saúde como um direito fundamental do cidadão e sob responsabilidade dos três entes da federação, compete aos Municípios, com apoio da União e do Estado, prestar atendimento de saúde (BRASIL, 1988). Apesar dessa prescrição legal, muitos municípios não comportam a estrutura necessária para prestar o atendimento devido. Em decorrência, seja por escassez de recursos financeiros, materiais ou humanos, a oferta do serviço público de saúde é insuficiente. É sob esse contexto que surgem os Consórcios Intermunicipais de Saúde (CIS), que representam, segundo Freitas, Oliveira e Cabral (2014), uma alternativa de organização e prestação de serviço público de saúde.

Os primeiros CIS surgem na década de 1990, a partir do processo de municipalização da saúde, que teve como início a promulgação da Constituição Federal de 1988 que, ao instituir a autonomia administrativa e alterar as relações entre os entes federados, deu poderes aos municípios de legislar também sobre o serviço público de atendimento à saúde (BASTOS, 2007). Conforme Ribeiro e Braga (2011) os CIS surgem com o objetivo de aproximar a gestão da saúde pública da população e melhorar a eficiência e a qualidade da aplicação dos recursos públicos na saúde da população.

Paula (2005), ao analisar o processo de redemocratização do Brasil e a eficiência do Estado em prover os serviços públicos, identifica a dimensão econômico-financeira como uma das que fundamentam a administração pública gerencial. A autora reconhece que o método de gestão e a clareza quanto à organização do Estado gerencialista revertem em eficiência do setor público, principalmente em aspectos econômico-financeiros.

De acordo com Prates (2012), cerca de $80 \%$ dos municípios brasileiros fazem parte de algum tipo de consórcio para prestação de serviços públicos. O autor destaca que a obtenção de ganhos de escala no acesso e uso de recursos financeiros, materiais e humanos, que dificilmente um município atingiria atuando isoladamente, é o principal benefício para os municípios que compõem os consórcios públicos. Na ótica de Coutinho (2006), viabilizar e agilizar projetos regionais, reduzindo a burocracia e compartilhando recursos, reduzir custos por meio de compras em maior escala, melhorar o acesso à saúde intermediando serviços, são objetivos dos consórcios. Neste sentido, Guimarães e Tavares (2012) destacam a demanda por maior profissionalização na gestão pública e propõem a adoção de ferramentas de apoio à gestão dos consórcios.

Dada a contextualização apresentada, o objetivo do estudo é identificar quais as práticas de gestão econômica e financeira são utilizadas por CIS estabelecidos no Rio Grande do Sul. Com essa identificação, analisada em relação ao que preceitua a literatura pertinente é possível aferir o grau de aderência dos CIS às práticas gerenciais recomendadas. 
A relevância do estudo está diretamente relacionada à sua importância social por identificar as práticas de gestão econômica e financeira que conduzem à eficiência, eficácia e economicidade da gestão pública. Cientificamente a justificativa ampara-se no interesse pelos consórcios intermunicipais de saúde, pois, como objeto de estudo, trata-se de uma estrutura recente e de interesse do meio acadêmico.

Este estudo se aproxima do realizado por Freitas, Oliveira e Cabral (2014) que investigou a atuação dos CIS, junto aos municípios da microrregião de Ubá, através de indicadores econômicos, sociais e de desenvolvimento da área da saúde. Entretanto, se diferencia por analisar a utilização de práticas de gestão econômica e financeira e CIS estabelecidos no Rio Grande do Sul.

Além desta introdução o artigo é composto por mais quatro seções. A segunda seção destina-se à apresentação do referencial teórico, a terceira dos aspectos metodológicos e a quarta pela apresentação e análise dos resultados. Por fim, tem-se as considerações finais do estudo, seguida das referências utilizadas no desenvolvimento de todo aporte teórico da pesquisa.

\section{REVISÃO DA LITERATURA}

\subsection{Gestão pública}

A gestão pública tem sido desafiada em trazer maior eficiência e eficácia nas suas ações e resultados. É nesse ambiente que surge a New Public Management (NPM), não necessariamente por trazer elementos de inovação, mas por contribuir com a indução de uma concepção mais econômica, financeira e gerencial da administração estatal. Assim, não existe uma convergência quanto aos padrões que conceituem ou caracterizem a NPM, pois estes variam conforme diferentes contextos administrativos (ORMOND, LÖFFLER, 1999). Estudos anteriores, tais como os de: Ormond e Löffler (1999); Jones e Thompson (2000); Pollitt e Bouckaert (2002); Kletz, Hénaut e Sardas (2014), identificam que o objetivo principal da NPM é a redução de custos de funcionamento dos setores estatais e a eficiência na prestação de serviços públicos.

A NPM prevê a divisão das estruturas hierárquicas burocráticas, descentralizando as decisões e atividades conforme as funções e objetivos a que estas se prestam. As atividades e funções passam a ser coordenadas por mecanismos de mercado, como sistemas comprador e fornecedor interno, terceirização, ou mesmo privatização total (ALMKLOV; ANTONSEN, 2014). Bresser-Pereira (1997), ao comentar os modelos de gestão pública, destaca algumas características básicas que diferenciam a administração pública gerencial frente à administração pública burocrática tradicional (Quadro 1).

\section{Quadro 1 - Características NPM frente administração pública burocrática}

\begin{tabular}{|l|l|}
\hline \multicolumn{1}{|c|}{ Administração Pública Gerencial (NPM) } & \multicolumn{1}{|c|}{ Administração Pública Burocrática } \\
\hline Orientada para o cidadão; & $\begin{array}{l}\text { Orientada para o processo legal, definição e execução } \\
\text { de procedimentos para contratação de pessoal; para } \\
\text { compra de bens e serviços; }\end{array}$ \\
\hline Foco na obtenção de resultados; & $\begin{array}{l}\text { Concentra-se nos processos para satisfazer as } \\
\text { demandas dos cidadãos sem considerar a alta } \\
\text { ineficiência envolvida; }\end{array}$ \\
\hline $\begin{array}{l}\text { Pressupõe que os políticos e os funcionários públicos } \\
\text { são merecedores de um grau real ainda que limitado } \\
\text { de confiança; }\end{array}$ & $\begin{array}{l}\text { Prefere prevenir estabelecendo estritos controles } \\
\text { legais; }\end{array}$ \\
\hline $\begin{array}{l}\text { Serve-se da descentralização e do incentivo à àn } \\
\text { criatividade e à inovação; }\end{array}$ & Controle dos procedimentos \\
\hline
\end{tabular}


PERFIL DA GESTÃO DE CONSÓRCIOS INTERMUNICIPAIS DE SAÚDE DO RIO GRANDE DO SUL: UM ESTUDO DAS PRÁTICAS DE GESTÃO ECONÔMICA E FINANCEIRA

Controle sobre os órgãos descentralizados através do contrato de gestão e avaliação de desempenho.
Os controles são preventivos, vem a priori. Definir indicadores de desempenho para as agências estatais é tarefa extremamente difícil.

Fonte: Adaptado de Bresser-Pereira (1997).

Pode-se entender a administração pública gerencial como evolução da administração burocrática e tendo, em seus primórdios, incentivo na redução dos custos da estrutura estatal e eficiência econômica na entrega dos serviços públicos. Também é identificado o foco da NPM, de acordo com Ormond, Löffler (1999), no desempenho da gestão governamental e da efetividade das políticas públicas. Assim, a NPM é reconhecida por fortalecer a capacidade de governo, principalmente relacionada a elaboração e execução de políticas públicas em atendimento as demandas da sociedade, por meio de uma concepção mais econômica, financeira e gerencial da administração estatal, promovendo o conceito de governança através de inovações gerenciais na área pública (MARINI; MARTINS, 2005). Oliveira, Silva e Bruni (2012) corroboram com esses princípios e destacam que a NPM surge em reconhecimento de que, em um ambiente de gestão efetiva os gestores passam a ter necessidade de instrumentos de gestão que forneçam informações gerenciais para sustentar o processo decisório em relação à aplicação dos recursos e desenvolvimento das políticas públicas.

\subsection{Consórcios Intermunicipais de Saúde}

Os primeiros Consórcios Intermunicipais de Saúde (CIS) no Brasil, surgiram na década de 1980. Tais experiências forneceram subsídios para a normatização dos CIS, promovendo-os como alternativa organizacional para os municípios na atuação da área da saúde (RIBEIRO; COSTA, 2000). A lei 8.080/1990, que regulamenta o SUS, prevê em seu artigo $10^{\circ}$ que "[...] os municípios poderão constituir consórcios para desenvolver em conjunto as ações e os serviços de saúde que lhes correspondam" (BRASIL, 1990).

O termo consórcio foi incorporado à Constituição Federal somente com a Emenda Constitucional 19/98 que incluiu o artigo 241 na Constituição de 1988 (BRASIL, 1998). Apesar disso, o regime jurídico consorcial no Brasil somente é admitido com a regulamentação da Lei $\mathrm{N}^{\mathrm{o}} 11.107 / 2005$, conhecida como Lei dos Consórcios Públicos, através do Decreto $\mathrm{n}^{\circ}$ 6.017/2007 (SILVA, 2008).

Bickers, Post e Stein (2009) argumentam que relações intergovernamentais regionais e metropolitanas ocorrem em busca de ganhos de eficiência para as jurisdições participantes. A crença é de que, em geral, os governos locais buscam parcerias regionais para prestação de bens e serviços no intuito de cortar custos ou obter ganhos de qualidade. Porém, para os autores, também existem as pressões e influências políticas motivadas pela maior visibilidade proporcionada por uma atuação regional para os políticos e partidos. Assim, percebe-se que diferentes objetivos, interesses, influências e ações de atores diversos agem sobre a atuação dos consórcios intermunicipais para prestação de bens e serviços em saúde.

\subsection{Gestão econômica e financeira}

A gestão econômica das organizações pode ser considerada como o foco principal dos estudos acerca de contabilidade gerencial. A contabilidade, no seu âmbito gerencial, conforme Atkinson et al. (2011), tem como propósito principal o melhor aproveitamento de recursos visando à otimização dos resultados. Para Assaf Neto (2003) a administração financeira desempenha basicamente quatro funções: (a) planejamento financeiro; (b) controle financeiro; (c) administração de ativos; e (d) administração de passivos.

Woon, Azizan e Samad (2011) destacam que a tomada de decisão financeira deve estar intimamente ligada aos fatores econômicos que norteiam o negócio e o mercado no qual este 
está inserido. Para que isto ocorra, se faz necessário que as organizações saibam quais são estes fatores e seus riscos inerentes e se posicionem perante o mercado, definindo os seus objetivos (econômicos e financeiros) e como pretende atingi-los.

Ferreira (2012) enfatiza que a gestão baseada em processos e informações é um dos fundamentos presentes na gestão pública de excelência. $\mathrm{O}$ autor admite que para buscar a eficiência e excelência, não basta simplesmente replicar as práticas de gestão próprias da iniciativa privada, um princípio da NPM. Para o mesmo, é necessário também considerar os aspectos da natureza da administração pública que as diferenciam da iniciativa privada, principalmente relacionada a resultados, maximização do bem-estar social frente à maximização do patrimônio individual, e a obrigação da continuidade dos serviços públicos.

Bomfin; Goulart e Melo (2012, p. 45) reconhecem a complexidade da estrutura administrativa e gestão dos CIS, a qual "exige do gestor um perfil empreendedor e habilidade de liderança que possa facilitar a articulação do órgão com as demandas políticas municipais e diminuir as dificuldades existentes". Os autores destacam que além desse perfil empreendedor e de liderança, o gestor público precisa de informações assertivas e tempestivas quanto às demandas e uso dos recursos disponíveis para fazê-lo.

\subsection{Práticas de gestão econômica e financeira}

Ao tratar da gestão econômica e financeira e das práticas necessárias para tal, Soutes (2006) faz referência aos artefatos da contabilidade gerencial como instrumentos de gestão. Soutes (2006, p. 23) detalha essas práticas como "[...] artefatos, atividades, ferramentas, filosofias de gestão, instrumentos, métodos de custeio, modelos de gestão, métodos de avaliação ou sistemas de custeio", os quais instrumentalizam a gestão de recursos e resultados. Para Atkinson et al. (2011) o objetivo da contabilidade de gestão, ou gerencial, é prover a administração com informações contábeis, financeiras e não financeiras que promovam o monitoramento, avaliação e controle do desempenho das organizações e apoiem o processo decisório. Entende-se, a partir de tais colocações, que os referidos artefatos se posicionam como práticas de gestão econômica e financeira, resumidos a seguir.

a) Planejamento estratégico: processo de definição de um empreendimento. É a visão abrangente do que se espera e de como alcançar os objetivos estabelecidos. A partir do seu detalhamento avaliam-se as possibilidades de execução e sua viabilidade econômica e financeira (MILLES; SNOW, 1978; KALD, NILSSON; RAPP, 2000).

b) Gestão estratégica de custos (GEC): uso das informações de custos para subsidiar o processo decisório (SOUZA; DIEHL, 2009); para Kumar e Shafabi (2011) a GEC não se limita a orientar o controle e corte de custos e destacam que ela é uma filosofia de criação de valor ao cliente ao menor custo, promoção da produtividade, maximização do lucro e satisfação do consumidor.

c) Planejamento operacional: para Ferreira e Diehl (2012), embora as limitações do enfoque financeiro, o planejamento contribui no processo de execução e controle; para os autores, as diferenças entre as diversas abordagens estão relacionadas ao nível estratégico, tático e operacional onde estão localizados os níveis de responsabilidades e processos orçamentários.

d) Execução e controle: Kald, Nilsson e Rapp (2000) destacam que os controles de gestão devem ser definidos a partir da estratégia da organização. Os autores propõem integrar os diferentes enfoques da teoria estratégica, compreendendo o padrão estratégico, a posição estratégica e a missão estratégica, em variáveis organizadas em uma estrutura única.

Referindo-se às práticas de gestão, Castro (2011) destaca que a administração pública deve trabalhar com base em planejamento e instrumentos que permitam sua execução e controle. Para o autor tais instrumentos têm por objetivo fornecer subsídios à tomada de decisão 
visando o planejamento, o controle da execução e a avaliação de desempenho dos programas e ações das entidades públicas conforme os princípios constitucionais.

Estudos nacionais sobre os CIS foram localizados, porém, a grande maioria deles trata do tema sob o foco dos CIS como instrumento para melhorar a performance na prestação de serviços públicos de saúde. Um estudo que se destaca com foco semelhante ao deste artigo, apesar de relacionado a consórcios públicos em geral e não aos CIS em particular, é o desenvolvido por Dallabrida e Zimermann (2009). Nesse estudo há destaque apenas ao uso do orçamento anual obrigatório por lei, não se utilizando de outras práticas de gestão econômica e financeira disponíveis. Não foram localizados estudos internacionais relacionados.

\section{PROCEDIMENTOS METODOLÓGICOS}

Dado o objetivo de conhecer as práticas de gestão econômica e financeira utilizadas por CIS, esta pesquisa se classifica como de natureza aplicada (GIL, 2010), pois contribui para gerar conhecimentos de aplicação prática para a solução de problemas específicos. Classificase também como descritiva por descrever as características de uma população - os CIS, e o fenômeno específico - o uso das práticas analisadas (GIL, 2010).

Quanto à abordagem trata-se de um estudo quantitativo, por fazer uso de técnicas da estatística descritiva como frequência relativa, média e desvio padrão, bem como técnicas exploratórias como correlação (CRESWELL, 2007). É Também qualitativo, dada a análise de significados dos achados. Quanto aos procedimentos técnicos empregados foi realizado levantamento através de pesquisa survey (COLLIS; HUSSEY, 2005).

A coleta dos dados foi realizada com uso de um instrumento elaborado sob o formato de escalas do tipo Likert com sete pontos. $\mathrm{O}$ instrumento foi estruturado em quatro blocos de proposições selecionadas para análise. Por meio desse instrumento foi possível apurar a concordância do respondente quanto à existência das ferramentas de gestão econômicofinanceira nos CIS e cálculo do ranking médio das respostas.

$\mathrm{O}$ instrumento de pesquisa, questionário, contém 21 questões e foi dividido em oito blocos: Motivos que levaram à formação do CIS; Planejamento Estratégico; Contabilidade Estratégica; Gestão Estratégica de Custos; Planejamento Operacional; Execução e Controle; Caracterização do CIS e; Caracterização do respondente.

O questionário foi submetido a pré-teste conforme o preconizado por Creswell (2007), realizado com dois gestores com experiência em consórcio e instituição pública de saúde. $\mathrm{O}$ primeiro gestor classificou o instrumento como "muito bom" e ponderou quanto a possíveis dificuldades por parte dos respondentes em relação a alguns termos técnicos. Em razão disso foram incluídas explicações nas proposições por blocos. O segundo gestor considerou as proposições como bem elaboradas dado o objetivo da pesquisa. Apenas propôs salientar nas escalas de resposta qual avaliação solicitada (níveis de concordância). O instrumento foi enviado aos respondentes via plataforma on line "Survey monkey" e precedido de contato telefônico.

A população da pesquisa foi definida a partir da listagem dos órgãos municipais auditados pelo Tribunal de Contas do Estado do Rio Grande do Sul e disponível para consulta em seu endereço eletrônico (RIO GRANDE DO SUL, 2014). A partir dessa lista identificou-se a existência de 19 CIS, dos quais 12 (63\%) participaram da pesquisa. 
Tabela 1 - Caracterização geral da amostra

\begin{tabular}{|c|c|c|c|c|c|}
\hline Nome Fantasia & $\begin{array}{l}\text { Município } \\
\text { Sede }\end{array}$ & $\begin{array}{l}\text { Número de } \\
\text { Municípios }\end{array}$ & $\begin{array}{c}\text { População } \\
\text { Abrangida (*) } \\
\text { (2013) }\end{array}$ & $\begin{array}{c}\text { PIB } \\
\text { Municípios } \\
(* *) \\
(\mathbf{2 0 1 1}) \\
\end{array}$ & $\begin{array}{c}\text { Ano de } \\
\text { Fundação }\end{array}$ \\
\hline COFRON & Santa Rosa & 25 & 245 & 5,298 & 1984 \\
\hline CI JACUI & Sobradinho & 12 & 186 & 3,161 & 1993 \\
\hline CIRC & Santa Maria & 31 & 558 & 4,716 & 1994 \\
\hline CONSISA VRT & Lajeado & 29 & 267 & 4,577 & 1995 \\
\hline CONSIM & Palmeira Missões & 17 & 106 & 1,974 & 1997 \\
\hline CIMAU Rodeio & Rodeio Bonito & 24 & 152 & 2,468 & 1998 \\
\hline COMAJA & Ibirubá & 29 & 283 & 7,254 & 2004 \\
\hline CIS CAI & Montenegro & 23 & 222 & 4,817 & 2004 \\
\hline CIS CENTRO SUL & Camaquã & 13 & 259 & 4,830 & 2005 \\
\hline CISGA & Garibaldi & 11 & 242 & 7,222 & 2006 \\
\hline COIS & São Luiz Gonzaga & 9 & 78 & 1,352 & 2006 \\
\hline CONISA & Nonoai & 12 & 81 & 1,785 & 2011 \\
\hline \multicolumn{2}{|c|}{ Totais } & 235 & 2.679 & 49,454 & \\
\hline
\end{tabular}

Fonte: dados da pesquisa.

Somente COFRON, CONSIM e COIS atuam exclusivamente na área da saúde. Todos os demais atuam em outras áreas de políticas públicas além da saúde (assistência social, meioambiente, turismo e desenvolvimento regional).

Os dados foram coletados no período de 05/11/2014 a 09/10/2014 e foram tabulados em planilha eletrônica conforme as proposições e valores atribuídos. A exemplo dos estudos de Fank, Angonese e Lavarda (2011) e Jordão et al. (2015), para análise dos dados foi utilizada a medição do Ranking Médio (RM), bem como a frequência em percentual, desvio padrão e correlação. Pelo RM se estabelece uma hierarquia dentre os CIS conforme a maior ou menor utilização das práticas de gestão analisadas. Com base nos valores e correlação estatística encontrados foram feitas inferências quanto à influência de variáveis como idade do consórcio, experiência dos gestores e nível de despesas na maior ou menor aderência dos CIS às práticas de gestão analisadas.

\section{APRESENTAÇÃO E ANÁLISE DOS DADOS}

\subsection{Perfis dos respondentes}

Apresenta-se no Quadro 2 os perfis dos respondentes representantes dos CIS pesquisados. Sobre tais perfis, Lorenzetti et al. (2014) argumentam que ainda prevalece no Brasil a cultura de que a administração é aprendida na prática e que qualquer profissional pode executá-la. Acrescentam que poucos gestores de instituições públicas estão devidamente habilitados para tal. Este argumento é pouco corroborado pelos dados desta pesquisa.

Quadro 2 - Perfis dos respondentes

\begin{tabular}{|l|c|l|c|}
\hline \multicolumn{1}{|c|}{ Cargo na Gestão } & Idade & \multicolumn{1}{|c|}{ Formação acadêmica } & $\begin{array}{c}\text { Tempo } \\
\text { (anos) no } \\
\text { atual cargo }\end{array}$ \\
\hline Procurador Jurídico & 44 & Direito & 17 \\
\hline Diretora Administrativa & 48 & Marketing e Esp. Gestão de Pessoas & NI \\
\hline Diretor Executivo & 73 & Bacharel em Administração & 8 \\
\hline Secretário Executivo & 59 & Gestão Hospitalar & 10 \\
\hline
\end{tabular}


PERFIL DA GESTÃO DE CONSÓRCIOS INTERMUNICIPAIS DE SAÚDE DO RIO GRANDE DO SUL: UM ESTUDO DAS PRÁTICAS DE GESTÃO ECONÔMICA E FINANCEIRA

\begin{tabular}{|l|c|l|c|} 
Coordenador Executivo & 59 & Ensino médio & 1 \\
\hline Diretor Executivo & 52 & - & 10 \\
\hline Secretário Executivo & 53 & Bacharel em Administração & 7 \\
\hline Cọ̣rdenadora & 39 & Gestão Pública & 2 \\
\hline Secretário Executivo & 48 & Superior incompleto em Administração & 2 \\
\hline Diretor Administrativo & 45 & Ciências Contábeis & 8 \\
\hline Secretário Executivo & 64 & Administração e Ciências Contábeis & 10 \\
\hline Diretor Executivo & 45 & Especialização em Gestão Pública & 20 \\
\hline
\end{tabular}

Fonte: dados da pesquisa.

Quanto à forma de nomeação, nove dos administradores executivos ocupam a função como cargo comissionado, portanto, por livre designação do conselho administrativo. Também constam dois funcionários cedidos por municípios e um terceiro contratado pelo regime da Consolidação das Leis do Trabalho. Conforme Ribeiro e Braga (2011), a Lei 11.107/2005 prevê que servidores podem ser cedidos pelos entes consorciados.

Observa-se experiência dos respondentes nos cargos de gestão ocupados, 67\% deles acima de sete anos. Conforme Silva et al. (2013), características individuais como tempo de experiência em cargos de gestão e vinculação ao setor público ou privado, influenciam na tomada de decisão.

\subsection{Perfis dos consórcios pesquisados}

O primeiro bloco de afirmativas trata dos motivos pelos quais os municípios associaram-se em consórcio. Quanto aos motivos para associação há muita semelhança entre os CIS. Os principais motivos são: redução de custos operacionais (RM 6,9), diversificação das especialidades $(6,7)$, compartilhamento de estrutura - profissionais, clínicas e hospitais (RM $6,7)$, capacitação técnica e de gestão em saúde (RM 5,9). Essas indicações corroboram o estudo de Costa (2009).

\subsection{Práticas de gestão econômica e financeira}

\subsubsection{Processo de planejamento}

O objetivo deste bloco é avaliar a adoção do Planejamento Estratégico por parte dos CIS e captar outras práticas relacionadas ao Processo de Planejamento destes. Dentre 12 respondentes, 10 concordam que desenvolvem suas atividades com base em planejamento estratégico, representando 83,3\%. Destes, $5(41,7 \%)$, concordam plenamente, alcançando uma média ponderada de 5,8. Apenas 1 respondente, discordou totalmente e 1, manteve-se neutro. A Tabela 2 descreve os resultados encontrados para as assertivas do bloco B, Planejamento Estratégico.

Tabela 2 - Planejamento estratégico

\begin{tabular}{|c|c|c|c|c|c|c|c|c|c|c|}
\hline \multirow{2}{*}{ Assertivas - Bloco B } & \multirow{2}{*}{ CIS } & \multicolumn{3}{|c|}{ Discordo } & \multirow{2}{*}{ Neutro: } & \multicolumn{3}{|c|}{ Concordo } & \multirow{2}{*}{$\mathbf{R M}$} & \multirow{2}{*}{ DP } \\
\hline & & 1 & 2 & 3 & & 5 & 6 & 7 & & \\
\hline $\begin{array}{l}\text { - As demandas e necessidades dos municípios } \\
\text { associados são informadas pelos próprios } \\
\text { municípios. }\end{array}$ & 12 & 0 & 0 & 0 & 1 & 0 & 1 & 10 & 6,67 & 0,89 \\
\hline $\begin{array}{l}\text { - O CIS desenvolve suas atividades com base } \\
\text { em Planejamento Estratégico. }\end{array}$ & 12 & 1 & 0 & 0 & 1 & 1 & 4 & 5 & 5,75 & 1,76 \\
\hline
\end{tabular}




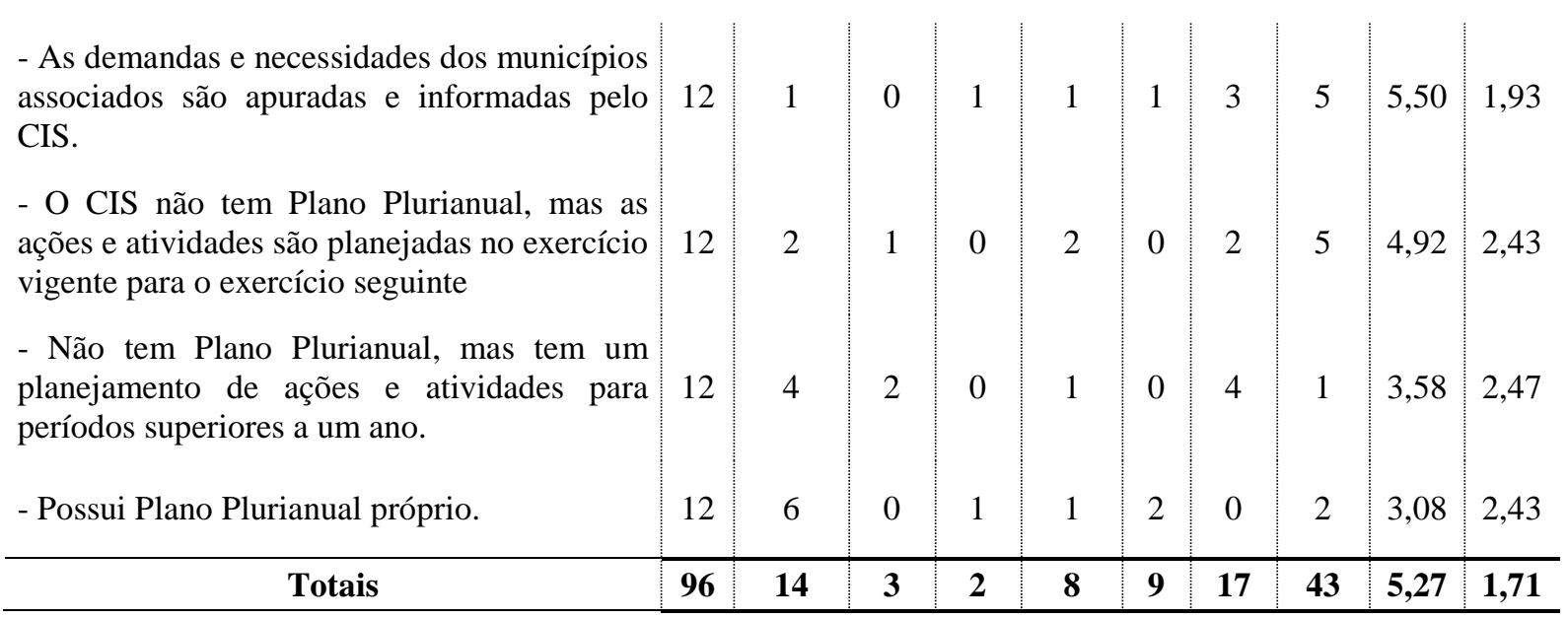

Fonte: dados da pesquisa.

Apesar da adesão ao uso do planejamento estratégico, 50\% dos CIS não adotam Plano Plurianual (PPA) próprio, reduzindo o RM para 3,08. Apesar do PPA ser um instrumento do planejamento estratégico do município (BRASIL, 2014), isso não impede os CIS de também terem o seu PPA. Assim, são os municípios que definem as diferentes políticas públicas, conforme suas estratégias de médio/longo prazo, cabendo aos CIS planejar a gestão destes recursos.

Em relação às demandas e necessidades dos municípios, e definição de metas e objetivos para os consórcios, foram apresentadas duas proposições: informadas pelos municípios ou pelos CIS. Para 10 dos 12 consórcios as demandas proveem dos municípios. Em relação à informação pelos CIS, embora apresente um RM de 5,50, há maior variabilidade nas escalas, refletido pelo maior DP (1,93 contra 0,89$)$. Esse resultado corrobora o estudo de Klering e Schröeder (2009), para os quais as estratégias são definidas pelos municípios.

\subsubsection{Gestão estratégica de custos}

Em relação às práticas de GEC as avaliações foram mais divergentes. As médias ponderadas ficaram abaixo de quatro, com 47 indicações abaixo do ponto neutro, chegando a $64 \%$ das respostas, conforme apresentado na Tabela 3.

Tabela 3 - Práticas de gestão estratégica de custos

\begin{tabular}{|c|c|c|c|c|c|c|c|c|c|c|}
\hline \multirow{2}{*}{ Assertivas - Detalhamento Bloco D } & \multirow{2}{*}{ CIS } & \multicolumn{3}{|c|}{ Discordo } & \multirow{2}{*}{\begin{tabular}{|c} 
Neutro \\
4
\end{tabular}} & \multicolumn{3}{|c|}{ Concordo } & \multirow{2}{*}{$\mathbf{R M}$} & \multirow{2}{*}{ DP } \\
\hline & & 1 & 2 & 3 & & 5 & 6 & 7 & & \\
\hline -Fatores determinantes de custo. & 12 & 4 & 0 & 1 & 1 & 2 & 3 & 1 & 3,83 & 2,33 \\
\hline -Custos Logísticos. & 12 & 5 & 0 & 0 & 1 & 2 & 3 & 1 & 3,67 & 2,46 \\
\hline -Gestão Baseada em Atividades (ABM). & 12 & 5 & 0 & 1 & 0 & 4 & 1 & 1 & 3,42 & 2,31 \\
\hline -Custo ciclo de vida dos produtos. & 12 & 5 & 0 & 1 & 3 & 1 & 2 & 0 & 3,08 & 2,02 \\
\hline -Custos intangíveis. & 12 & 7 & 0 & 1 & 0 & 1 & 2 & 1 & 2,83 & 2,44 \\
\hline -Custo Total de Uso e Propriedade. & 12 & 7 & 0 & 0 & 1 & 4 & 0 & 0 & 2,58 & 1,98 \\
\hline -Custo Kaizen - melhoria contínua. & 12 & 9 & 0 & 1 & 1 & 0 & 1 & 0 & 1,83 & 1,64 \\
\hline Totais & 84 & 42 & $\mathbf{0}$ & 5 & 7 & 14 & 12 & 4 & 3,04 & 2,17 \\
\hline
\end{tabular}

Fonte: dados da pesquisa.

Neste bloco foi também proposta uma questão aberta: Você entende que o CIS utiliza práticas de GEC? Alguns comentários extraídos apresentam: "estamos em fase de análise"; um dos respondentes apontou que o CIS não está preparado para usar a GEC e complementa que "é uma ferramenta muito pouco usada nos órgãos públicos"; teve-se também a afirmativa de que "a atividade do consórcio não está relacionada à GEC". 
O estudo de Mauss e Souza (2008) constatou a ausência de uma cultura de gestão de custos na administração pública brasileira, porém, nesta pesquisa a preocupação com a redução de custos aparece em primeiro lugar dentre os motivos de associação. Alemão, Gonçalves e Drumond (2013) também apontam a falta de informação de custos padronizada e apropriada para o processo decisório na área da saúde.

\subsubsection{Planejamento operacional}

A

Tabela 4 apresenta as avaliações referentes às práticas relacionadas ao orçamento operacional. Há um RM elevado na adoção geral do planejamento operacional, porém, à medida que se detalha a utilização nota-se acentuadas quedas nesse RM. Há também desvios padrões significativos sobre à utilização das peças orçamentárias, indicando que não há um padrão na adoção do orçamento como ferramenta gerencial.

Tabela 4 - Planejamento operacional

\begin{tabular}{|c|c|c|c|c|c|c|c|c|c|c|}
\hline \multirow{3}{*}{ Assertivas - Bloco E } & \multirow{3}{*}{ CIS } & \multicolumn{3}{|c|}{ Discordo } & \multirow{3}{*}{\begin{tabular}{|c|} 
Neutro \\
4 \\
\end{tabular}} & \multicolumn{3}{|c|}{ Concordo } & \multirow{3}{*}{ RM } & \multirow{3}{*}{ DP } \\
\hline & & & & & & & & & & \\
\hline & & 1 & 2 & 3 & & 5 & 6 & 7 & & \\
\hline $\begin{array}{l}\text {-Utiliza Orçamento Operacional como } \\
\text { instrumento de gestão. }\end{array}$ & 12 & 0 & 0 & 1 & 0 & 1 & 3 & 7 & 6,25 & 1,22 \\
\hline $\begin{array}{l}\text {-O orçamento é revisado durante o } \\
\text { período de execução das atividades. }\end{array}$ & 12 & 0 & 0 & 0 & 3 & 0 & 2 & 7 & 6,08 & 1,31 \\
\hline $\begin{array}{l}\text {-O presidente e o conselho cobram } \\
\text { objetivos estabelecidos. }\end{array}$ & 12 & 0 & 0 & 2 & 2 & 1 & 2 & 5 & 5,50 & 1,62 \\
\hline $\begin{array}{l}\text {-Utiliza Demonstração do Resultado } \\
\text { projetada e real. }\end{array}$ & 12 & 2 & 0 & 0 & 1 & 1 & 1 & 7 & 5,50 & 2,32 \\
\hline $\begin{array}{l}\text {-Utiliza Orçamento de Capital } \\
\text { (Imobilizações). }\end{array}$ & 12 & 2 & 0 & 1 & 0 & 1 & 2 & 6 & 5,33 & 2,35 \\
\hline $\begin{array}{l}\text {-Utiliza Balanço Patrimonial projetado e } \\
\text { real. }\end{array}$ & 12 & 3 & 0 & 1 & 0 & 0 & 2 & 6 & 5,00 & 2,66 \\
\hline $\begin{array}{l}\text {-Utiliza Orçamento de Caixa projetado e } \\
\text { real. }\end{array}$ & 12 & 2 & 1 & 0 & 3 & 0 & 1 & 5 & 4,75 & 2,42 \\
\hline Totais & 84 & 9 & 1 & 5 & 9 & 4 & 13 & 43 & 5,49 & 1,98 \\
\hline
\end{tabular}

Fonte: dados da pesquisa.

Em questão aberta foi perguntado se o CIS utiliza efetivamente o orçamento como ferramenta de gestão, para apenas 5 de 12 respondentes a utilização é intensa. Os demais responderam que usam em parte ou que usam somente para atender a legislação. Ferreira e Diehl (2012) destacam a importância, no planejamento estratégico, do orçamento como desdobramento e comunicação deste. Considerando que 10 dos 12 CIS declararam trabalhar com base em planejamento estratégico, parece haver maior resistência em usar o orçamento para este fim.

\subsubsection{Execução e controle}

O elemento de maior escore identificado foi Controles Internos (CI). Tal resultado contraria o estudo de Reis e Diehl (2015) que identificaram em sítios dos CIS os CI como o item de governança menos evidenciado. Apenas o consórcio CONISA apontou não praticá-lo. Este CIS atribuiu escore 1 a todas as práticas deste bloco e apenas presta contas para o Tribunal 
de Contas do Estado e para os municípios participantes, uma obrigatoriedade legal. A Tabela 5 descreve os resultados encontrados para o bloco $\mathrm{F}$, de execução e controle.

Tabela 5 - Execução e controle

\begin{tabular}{l|c|c|c|c|c|c|c|c|c|c|c}
\hline \multicolumn{1}{c}{ Assertivas - Bloco F } & CIS & \multicolumn{3}{c|}{ Discordo } & Neutro & \multicolumn{3}{c|}{ Concordo } & RM & DP \\
\hline - Controles internos & & $\mathbf{1}$ & $\mathbf{2}$ & $\mathbf{3}$ & $\mathbf{4}$ & $\mathbf{5}$ & $\mathbf{6}$ & $\mathbf{7}$ & & \\
- Gestão do ativo fixo & 12 & 1 & 0 & 0 & 0 & 0 & 2 & 9 & 6,33 & 1,72 \\
- Fluxo de caixa & 12 & 1 & 1 & 0 & 2 & 0 & 2 & 6 & 5,42 & 2,15 \\
- Auditoria Interna & 12 & 2 & 0 & 0 & 1 & 1 & 2 & 6 & 5,42 & 2,27 \\
- Sistemas de Informações Gerenciais & 12 & 2 & 0 & 0 & 2 & 0 & 1 & 7 & 5,42 & 2,35 \\
- Indicadores financeiros & 12 & 1 & 0 & 0 & 2 & 3 & 3 & 3 & 5,25 & 1,71 \\
- Gestão de Tesouraria & 12 & 1 & 0 & 0 & 4 & 1 & 3 & 3 & 5,08 & 1,78 \\
- Indicadores não financeiros & 12 & 4 & 0 & 0 & 1 & 0 & 4 & 3 & 4,42 & 2,64 \\
\hline \multicolumn{1}{r|}{ Totais } & 12 & 4 & 0 & 0 & 4 & 3 & 1 & 0 & 3,42 & 1,88 \\
\hline
\end{tabular}

Fonte: dados da pesquisa

A adoção de práticas de execução e controle obteve 63 escores positivos de um total de 96 possíveis, representando 65\%. Porém, pelos desvios padrões apurados verifica-se heterogeneidade do grau de concordância quanto à adesão às práticas de execução e controle, com certa polarização dos escores obtidos se considerado o ponto neutro e o escore 1. O baixo escore alcançado pelo item Indicadores não Financeiros sinaliza que a ênfase dos CIS está mais na gestão dos recursos financeiros e cumprimento das metas orçamentárias, do que na efetividade da prestação do serviço em saúde.

\subsection{Ranking médio dos consórcios}

A partir dos escores atribuídos a cada proposição foi possível estabelecer um ranking para os CIS classificando-os conforme as práticas de gestão econômica e financeira utilizadas, na percepção dos seus gestores. A Tabela 6 apresenta a frequência absoluta dos escores atribuídos pelos respondentes, a média e desvio padrão para cada CIS. A sequência de apresentação foi estabelecida pela ordenação dos CIS pela média dos escores atribuídos.

Tabela 6 - Ranking médio dos consórcios

\begin{tabular}{|c|c|c|c|c|c|c|c|c|c|}
\hline \multirow{2}{*}{ CIS } & \multicolumn{3}{|c|}{ Discordo } & \multicolumn{2}{|c|}{ Neutro } & \multicolumn{2}{|c|}{ Concordo } & \multirow{2}{*}{ Média } & \multirow{2}{*}{$\begin{array}{l}\text { Desvio } \\
\text { Padrão }\end{array}$} \\
\hline & 1 & 2 & 3 & 4 & 5 & 6 & 7 & & \\
\hline CIMAU Rodeio & 1 & 0 & 0 & 2 & 4 & 7 & 20 & 6,21 & 1,28 \\
\hline CI Jacuí & 0 & 0 & 0 & 0 & 7 & 19 & 8 & 6,03 & 0,66 \\
\hline CIS Caí & 3 & 1 & 2 & 3 & 4 & 11 & 10 & 5,26 & 1,85 \\
\hline CONSISA VRT & 6 & 0 & 0 & 6 & 2 & 5 & 15 & 5,15 & 2,21 \\
\hline CISGA & 5 & 1 & 0 & 1 & 7 & 14 & 6 & 5,06 & 1,94 \\
\hline COMAJA & 5 & 0 & 1 & 4 & 9 & 7 & 8 & 4,91 & 1,93 \\
\hline CIRC & 8 & 0 & 0 & 12 & 0 & 0 & 14 & 4,24 & 2,20 \\
\hline CONSIM & 12 & 0 & 0 & 3 & 0 & 5 & 14 & 4,53 & 2,35 \\
\hline COFRON & 7 & 0 & 6 & 8 & 0 & 4 & 9 & 4,47 & 2,69 \\
\hline CI Centro-Sul & 7 & 0 & 1 & 21 & 4 & 1 & 0 & 3,53 & 1,38 \\
\hline COIS & 20 & 0 & 0 & 2 & 0 & 0 & 12 & 3,29 & 2,82 \\
\hline CONISA & 24 & 0 & 0 & 1 & 3 & 1 & 5 & 2,47 & 2,35 \\
\hline Amostra & 98 & 2 & 10 & 63 & 40 & 74 & 121 & 4,60 & 2,31 \\
\hline
\end{tabular}

Fonte: dados da pesquisa.

Análise específica mostra que, dentre os CIS pesquisados, o CIMAU Rodeio aparece como o de melhor média nos escores atribuídos pela avaliação dos respondentes. $\mathrm{O}$ 
item com avaliação mais baixa foi Análise de Ponto de Equilíbrio, com escore 1. Esse CIS posiciona-se neutro para dois itens, Custo Kaizen e Técnicas de Análise sobre Investimentos. O CIMAU é o mais antigo, constituído em 1984; o respondente está há 10 anos no cargo. Esses são dois fatores que podem contribuir para a maturidade da organização, quando novas técnicas vão sendo incorporadas, o aprendizado se consolida e pela experiência mais longeva que pode influenciar o respondente pela crença de seu domínio de técnicas gerenciais, mesmo que não se reflitam plenamente na prática.

O segundo colocado é o CI Jacuí, com média de 6,03 e desvio padrão de 0,66, o menor da amostra. De qualquer forma, entende-se que este consórcio faz uso, em maior ou menor grau, das práticas analisadas. O CIS que aponta menor utilização das práticas é o Conisa, Com RM de apenas 2,5 e desvio padrão de 2,35, o segundo mais elevado.

Pelos dados analisados verifica-se grande disparidade entre os CIS em relação às suas características de gestão. Estes achados estão alinhados com os estudos de Luque et al. (2008), Souza (2009) e Souza, Ortiz e Almeida (2013) e, que verificaram a necessidade de se desenvolver e aprimorar a cultura de uso gerencial das técnicas de contabilidade e, principalmente, gestão de custos por sua importância para a gestão econômica e financeira.

\subsection{Análise estatística exploratória}

\subsubsection{Correlação entre perfil e variáveis dos CIS}

Identificadas as práticas e classificados os CIS pelo grau de utilização, algumas correlações foram testadas frente aos RM. Visa-se procurar variáveis que possam explicar os escores e a classificação referente às práticas de gestão econômica e financeira analisadas. Gray (2014) propõe a seguinte escala de força de correlação: de 0,10 a 0,29 , pequena correlação, de 0,30 a 0,49, média correlação e de 0,50 a 1,00, grande correlação. Os dados da Tabela 7 indicam que a variável ano de constituição do CIS não tem nenhuma correlação com o Ranking Médio, contrariando o estudo de Ikäheimo e Taipaleenmäki (2010) que supõe que com o tempo a organização tende a incorporar novas práticas e procedimentos em sua gestão, apesar do estudo citado ter sido realizado no contexto industrial.

Tabela 7 - Correlação RM x antiguidade do CIS e experiência do gestor

\begin{tabular}{|c|c|c|c|c|c|}
\hline CIS & $\mathbf{R M}$ & $\begin{array}{c}\text { Ano de } \\
\text { constituição }\end{array}$ & $\begin{array}{c}\text { Idade do Gestor } \\
\text { Principal }\end{array}$ & $\begin{array}{l}\text { Tempo no atual } \\
\text { cargo/função }\end{array}$ & $\begin{array}{c}\text { Tempo de } \\
\text { experiência na } \\
\text { área }\end{array}$ \\
\hline CIMAU Rodeio & 6,21 & 1.984 & 59 & 10 & 10 \\
\hline CI Jacuí & 6,03 & 2.004 & 52 & 10 & 15 \\
\hline CIS Caí & 5,26 & 2.006 & 73 & 8 & 8 \\
\hline CONSISA VRT & 5,15 & 2.004 & 48 & 0 & 10 \\
\hline CISGA & 5,06 & 2.011 & 45 & 3 & 20 \\
\hline COMAJA & 4,91 & 1.998 & 45 & 8 & 8 \\
\hline CIRC & 4,53 & 1.993 & 59 & 1 & 20 \\
\hline CONSIM & 4,47 & 2.006 & 53 & 7 & 7 \\
\hline COFRON & 4,24 & 1.994 & 44 & 17 & 17 \\
\hline CI CENTRO-SUL & 3,53 & 2.005 & 48 & 0 & 12 \\
\hline COIS & 3,29 & 1.995 & 64 & 10 & 10 \\
\hline CONISA & 2,47 & 1.997 & 39 & 2 & 2 \\
\hline Correlação & & $-0,0009$ & $\mathbf{0 , 3 0 8 3}$ & 0,2456 & $\mathbf{0 , 3 3 2 1}$ \\
\hline Média & & 2.000 & 52 & 6 & 12 \\
\hline Desvio Padrão & & 7,24 & $\mathbf{9 , 3 3}$ & 4,92 & 5,24 \\
\hline
\end{tabular}

Fonte: dados da pesquisa 
Quanto à idade e tempo de experiência do gestor, verifica-se uma média correlação com o grau de adoção das práticas de gestão. Há, portanto, corroboração parcial com Liguori e Steccolini (2012), os quais destacam a importância do papel de liderança na condução de mudanças e adoção de novas práticas de gestão.

Tabela 8 - Despesas dos CIS

\begin{tabular}{|c|c|c|c|c|}
\hline CIS & $\mathbf{R M}$ & $\begin{array}{c}\text { Despesas Total } \\
(2013)(*)\end{array}$ & $\begin{array}{c}\text { Despesas Saúde } \\
(2013)(*)\end{array}$ & $\begin{array}{c}\text { Despesas } \\
\text { Adm/Manut } \\
(2013)(*)\end{array}$ \\
\hline CIMAU Rodeio & 6,21 & 6.097 & 5.668 & 186 \\
\hline CI Jacuí & 6,03 & 4.809 & 4.170 & 467 \\
\hline CIS Caí & 5,26 & 12.069 & 11.186 & 483 \\
\hline CONSISA VRT & 5,15 & 6.713 & 6.056 & 465 \\
\hline CISGA & 5,06 & NI & NI & NI \\
\hline COMAJA & 4,91 & 6.050 & 4.483 & 372 \\
\hline CIRC & 4,53 & 4.186 & 3.567 & 576 \\
\hline CONSIM & 4,47 & NI & NI & NI \\
\hline COFRON & 4,24 & 4.165 & 3.931 & 234 \\
\hline CI CENTRO-SUL & 3,53 & 1.340 & 1.067 & 174 \\
\hline COIS & 3,29 & 3.224 & 3.064 & 160 \\
\hline CONISA & 2,47 & 1.764 & 1.498 & 265 \\
\hline Correlação & & 0,6365 & 0,6157 & 0,4263 \\
\hline \multicolumn{5}{|c|}{ Legenda: (*) Valores em milhares. } \\
\hline
\end{tabular}

Fonte: dados da pesquisa

Em relação ao volume de recursos movimentados, a Tabela 8 apresenta os valores das despesas dos CIS conforme demonstrativos contábeis dos consórcios divulgados pelo Tribunal de Contas do Estado do Rio Grande do Sul. Percebe-se uma grande variação da proporção das despesas pela população total abrangida. Uma dificuldade encontrada quanto à estes valores é a identificação da destinação dos gastos, o que impede maiores inferências quanto à aplicação dos recursos.

Para as variáveis despesas totais e despesas com saúde, mostradas na Tabela 8, é possível afirmar que existe grande correlação com a adoção das práticas de gestão, dado os respectivos coeficientes de 0,636 e 0,615, indicando que elas podem induzir a maior aplicação dessas práticas. Assim, consórcios que realizam maiores despesas e, provavelmente, tem acesso a maiores recursos, tendem a ter maior preocupação com a gestão destes recursos.

\section{CONSIDERAÇÕES FINAIS}

Este artigo analisou o uso de práticas de gestão econômica e financeira por consórcios intermunicipais de saúde (CIS) do Rio Grande do Sul (RS). Foram pesquisados 12 dos 19 CIS existentes. Os dados foram coletados por instrumento de pesquisa respondidos por representantes da gestão dos referidos CIS.

Identificou-se que os CIS se associam tendo por objetivos principais a redução de custos e o compartilhamento e ampliação das estruturas municipais de saúde. Dentre os quatro grupos analisados, o que apresentou maior aderência às práticas verificadas foram, pela ordem, o planejamento operacional (RM 5,5), Gestão Estratégica de custos (RM 5,7), planejamento estratégico (RM 5,3), execução e controle (RM 5,1) e contabilidade estratégica (RM 4,3). Observa-se que há ainda, em todos eles, um considerável caminho para evoluir visando dotar os CIS de uma maior adoção das práticas de gestão recomendadas pela literatura.

Também se constatou, por meio da técnica de correlação estatística que a dimensão das despesas e experiência dos gestores são os fatores mais relacionados (média e alta, 
respectivamente) ao uso das práticas analisadas. Resultado contrário é encontrado quando a variável considerada é o tempo de existência dos CIS, cuja correlação mostra-se pequena.

Embora não se tenha avaliado o desempenho econômico dos CIS, tendo em vista a não disponibilidade de dados para análise e também por não ser este o foco principal do estudo, considera-se que a pesquisa alcançou seu objetivo de traçar o perfil dos CIS quanto à utilização de práticas de gestão econômica e financeira.

A adesão dos municípios aos consórcios busca reduzir os custos e despesas pelo ganho de escala dada a atuação conjunta. De outra parte, os resultados sinalizam que a preocupação precípua da gestão dos CIS é voltada para o cumprimento estrito das obrigações legais e normativas para si e para os municípios. Portanto, para os CIS o foco de cliente parece estar no município e aspectos legais, e não no usuário do serviço de saúde.

Dados os resultados da pesquisa, e visando aprofundar o conhecimento sobre aspectos específicos da gestão econômica e financeira dos CIS, sugere-se o desenvolvimento de estudos de caso nos dois CIS com maior aderência às práticas pesquisadas e analisá-los comparativamente aos dois CIS com menor aderência. Acredita-se que tal comparabilidade poderá acrescentar novas e interessantes descobertas sobre o tema.

\section{REFERÊNCIAS}

ALEMÃO, M. M.; GONÇALVES, M. A.; DRUMOND, H. A. Estudo da utilização da informação de custos como ferramenta de gestão em organização pública: o estudo do SIGHcustos. Perspectivas em Gestão \& Conhecimento. Paraíba, v. 3, n. 1, p. 210-226, 2013.

ALMKLOV, P. G.; ANTONSEN, S. Making work invisible: new public management and operational work in critical infrastructure sectors. Public Administration, v. 92, n. 2, p. $477-$ 492, 2014.

ASSAF NETO, A. Finanças corporativas e valor. São Paulo: Atlas, 2003.

ATKINSON, A.A.; BANKER, R.D.; KAPLAN, R.S.; YOUNG, S.M. Contabilidade gerencial. 3. ed. São Paulo, Atlas, 2011.

BASTOS, F.A. Os consórcios intermunicipais e a nova legislação. Polémica Revista Eletrônica, Rio de Janeiro, v. 19, p. 1-12, jan./mar., 2007.

BICKERS, K.; POST, S.; STEIN, R. The political market for intergovernmental cooperation. Cambridge: Cambridge University Press, 2009.

BOMFIN, D. F.; GOULART, I. B.; MELO, O. R. Ações de aprendizagem e liderança em consórcios da área de saúde. Revista Economia e Gestão, Belo Horizonte v. 12, n. 29, p. 42 $62,2012$.

BRASIL. Constituição de 1988. Constituição da República Federativa do Brasil: promulgada em 5 de outubro de 1988. Diário Oficial da União, Brasília, 05 out. 1988.

BRASIL. Lei 8.080, de 19 de setembro de 1990. Dispõe sobre as condições para a promoção, proteção e recuperação da saúde, a organização e o funcionamento dos serviços correspondentes e dá outras providências. Diário Oficial da União, Brasília, 20 set. 1990. 
BRASIL. MINISTÉRIO DA SAÚDE. Departamento de Informática do SUS. Cadastro Nacional de Estabelecimentos de Saúde - CNES. Brasília, 2014. Disponível em: <http://cnes.datasus.gov.br/Index.asp?home=1>. Acesso em: 03 jul. 2014.

BRESSER-PEREIRA, L. C. Estratégia e estrutura para um novo Estado. Revista de Economia Política, São Paulo, v. 17, n. 3, p. 24-38, 1997.

CASTRO, D. P. Auditoria, contabilidade e controle interno no setor público. 4. ed. São Paulo, 2011.

COLLIS, H.; HUSSEY, R. Pesquisa em administração. 2, ed. Porto Alegre: Bookman, 2005.

COSTA, W. H. A. O papel dos consórcios intermunicipais de saúde na retomada do desenvolvimento econômico e social. In: CONGRESSO NACIONAL DO CONPENDI, 18, 2009, São Paulo. Anais... São Paulo, 2009. p.10768-10778.

COUTINHO, F. M. A. Os consórcios públicos como instrumento potencializador de políticas públicas. In: ENCONTRO DA ASSOCIAÇÃO NACIONAL DE PÓS-GRADUAÇÃO E PESQUISA EM ADMINISTRAÇÃO, 30, Salvador, 2006. Anais... ANPAD: Salvador, 2006.

CRESWELL, J. W. Projeto de pesquisa: métodos qualitativo, quantitativo e misto. 2. ed. Porto Alegre, Artmed, 2007.

DALLABRIDA,V.; ZIMERMANN,V. Descentralização na gestão pública e estruturas subnacionais de gestão do desenvolvimento: o papel dos consórcios intermunicipais. Revista Brasileira de Gestão e Desenvolvimento Regional, Taubaté, v .5, n. 3, p. 3-28, 2009.

FANK, O. L.; ANGONESE, R.; LAVARDA, C. E. F. A percepção dos gestores acadêmicos de uma IES quanto às críticas ao orçamento. Contabilidade, Gestão e Governança. Brasília v. 14, n. 1, p. 82-93, 2011.

FERREIRA, A. R. Modelo de excelência em gestão pública. Revista Eixo, Brasília, v.1, n. 1, p. 31-43, 2012.

FERREIRA, F.B.; DIEHL, C.A. Orçamento empresarial e suas relações com o planejamento estratégico. Revista Pensar Contábil, Rio de Janeiro, v.14, n. 54, p. 46-57, 2012.

FREITAS, B. R.; OLIVEIRA, A. R.; CABRAL, K. F. D. Consórcios intermunicipais de saúde como instrumentos de cooperação para os municípios da microrregião de Ubá. In: ENCONTRO NACIONAL DE PESQUISADORES EM GESTÃO SOCIAL, 8, 2014, Cachoeira. Anais... ENAPEGS: Cachoeira, 2014.

GIL, A. C. Como elaborar projetos de pesquisa. 5. ed. São Paulo: Atlas, 2010.

GRAY, D. E. Pesquisa no mundo real. 2. ed. Porto Alegre, Bookman, 2014.

GUIMARÃES, J.C.C.C.; TAVARES, M. C. O BSC e a administração dos consórcios intermunicipais de saúde: um estudo de caso sobre sua aplicabilidade. Revista IberoAmericana de Estratégia (RIAE), São Paulo, v. 11, n. 3, p.234-262, 2012. 
IKÄHEIMO, S.; TAIPALEENMÄKI, J. The divergence and convergence of financial accounting and Institutional analysis of the U.S., Germany and Finland. Die Betriebswirtschaft, v. 70, n. 4, p. 349-368, 2010.

JONES, L. R.; THOMPSON, F. Um modelo para a nova gerência pública. Revista do Serviço Público, Brasília, v. 51, n. 1, p. 41-80, 2000.

JORDÃO, R. V. D.; PELEGRINI, F. G.; JORDÃO, A. C. T.; JEUNON, E. E.; Fatores críticos na gestão de projetos: um estudo de caso numa grande empresa latino-americana de classe mundial. Gest. Prod., São Carlos, v. 22, n. 2, p. 280-294, 2015.

KALD, M.; NILSSON, F.; RAPP, B. On strategy and management control: The importance of classifying the strategy of the business. British Journal of Management, n. 11, p. 197-212. 2000 .

KLERING, L.; SCHRÖEDER, C. S. Políticas e estratégias públicas municipais: novos desafios e perspectivas para o desenvolvimento local. Redes, Santa Cruz do Sul, v. 13, n. 2, p. 144-160, 2009.

KLETZ, F.; HÉNAUT, L.; SARDAS, J.C. New public management and the professions within cultural organizations: one hybridization may hide another. International Review of Administrative Sciences, v. 80, n. 1, p. 89-109, 2014.

KUMAR, A.; SHAFABI. Strategic cost management - suggested framework for 21st Century. Journal of Business and Retail Management Research, v. 5, n. 2, p. 118-131, 2011.

LIGUORI, M.; STECCOLINI, I. Accounting change: explaining the outcomes, interpreting the process. Accounting, Auditing \& Accountability Journal, v. 25, n. 1, p. 27-70, 2012.

LORENZETTI, J.; LANZONI, G. M. DE M.; ASSUIT, L. F. C.; PIRES, E. D. P.; RAMOS, F. R. S. Gestão em saúde no Brasil: diálogo com gestores públicos e privados. Texto \& Contexto - Enfermagem, Florianópolis, v. 23, n. 2, p. 417-425, 2014.

LUQUE, C. A.; CRUZ, H. N.; AMARAL, C. M.; BENDER, S.; SANTOS, P. M. DOS. O processo orçamentário e a apuração de custos de produtos e serviços no setor público do Brasil. Revista do Serviço Público, Brasília, v. 59, n. 3, p. 309-331, 2008.

MARINI, C.; MARTINS, H. Um modelo de gestão governamental para resultados. In: SEMINÁRIO DE ADMINISTRAÇÃO PÚBLICA, 1, Brasília, 2005. Anais... Brasília, 2005.

MAUSS, C.V.; SOUZA, M.A. Gestão de custos aplicada ao setor público: modelo para mensuração e análise da eficiência e eficácia governamental. São Paulo, Atlas, 2008.

MILLES, R. E.; SNOW C.C. Organizational strategy. McGraw-Hill, New York. 1978.

OLIVEIRA, A. B. R.; SILVA, U. B.; BRUNI, A. L. Gerencialismo e desafios contemporâneos da gestão dos custos públicos no Brasil. Revista de Estudos Contábeis, Londrina, v. 3, n. 5, p. 63-82, 2012. 
ORMOND, D.; LÖFFLER, E. A nova gerência pública. Revista do Serviço Público, v. 50, n. 2, p. 66-96, 1999.

PAULA, A.P.P. Administração pública brasileira entre o gerencialismo e a gestão social. RAERevista de Administração de Empresas, Rio de Janeiro, v.45, p.36-49, 2005.

PRATES, A.M.Q. Federalismo no Brasil: os consórcios públicos intermunicipais no período recente, 2012. Tese (Doutorado em Economia). Unicamp, Campinas, 2012.

POLLITT, C.; BOUCKAERT, G. Avaliando reformas da gestão pública: uma perspectiva internacional. Revista do Serviço Público, Brasília, v. 53, n. 3, p. 7-32, 2002.

REIS, H. C.; DIEHL, C. A. A Governança corporativa em consórcios intermunicipais públicos de saúde no Rio Grande do Sul. Revista Eletrônica Gestão \& Saúde, v. 6, Supl. 3, Jun., p.2162-97, 2015.

RIBEIRO, H. A.; BRAGA, R.Q. Administração de consórcios intermunicipais de saúde. Revista Brasileira de Gestão e Engenharia, São Gotardo, n. 3, p. 105-122, 2011.

RIBEIRO, J. M.; COSTA, N. R. Regionalização da assistência à saúde no brasil: os consórcios municipais no sistema único de saúde (SUS). Planejamento e Políticas Públicas. Rio de Janeiro. v. 22, p. 173-220. 2000.

RIO GRANDE DO SUL. TRIBUNAL DE CONTAS DO ESTADO DO RS. Instrução Normativa $\mathrm{n}^{\circ} 7$ de 25 de abril de 2014. Dispõe sobre a forma de publicação das informações do Relatório Resumido da Execução Orçamentária (RREO) e do Relatório de Gestão Fiscal (RGF) Diario Eletrônico do TCERS, Porto Alegre, 30 abr. 2014.

SILVA, C. D. O. Os consórcios públicos e a efetividade dos direitos fundamentais à saúde e ao meio ambiente ecologicamente equilibrado. 2008. Mestrado (Direito). PUC-RS. Porto Alegre, 2008.

SILVA, U. B.; BRUNI, A. L.; DIAS_FILHO, J. M.; SANTOS, E. B. Decisões, complexidades, agentes e sunk costs: um estudo comparativo envolvendo gestores com diferentes perfis. ABCustos, São Leopoldo, v. 8, n. 2, 2013.

SOUTES, D. O. Uma investigação do uso de artefatos da contabilidade Gerencial por empresas brasileiras. 2006. Dissertação (Mestrado em Ciências Contábeis) Universidade de São Paulo, São Paulo, 2006.

SOUZA, P. C. Avaliação econômica em saúde: aplicação da análise custo-volume-superávit para avaliar hospitais públicos. In: ENCONTRO NACIONAL DE ENGENHARIA DE PRODUÇÃO, 29, 2009. Salvador. Anais... ABEPRO: Salvador, 2009.

SOUZA, M. A.; DIEHL, C. A. Gestão de custos: uma abordagem integrada entre contabilidade, engenharia e administração. São Paulo: Atlas, 2009.

SOUZA, M. A.; ORTIZ, A. C. S.; ALMEIDA, L. B. Sistema de informações gerenciais de controladoria em órgãos públicos: um estudo nos municípios mato-grossenses da região sudoeste. In: ENCONTRO DA ASSOCIAÇÃO NACIONAL DE PÓS-GRADUAÇÃO E 
PESQUISA EM ADMINISTRAÇÃO, 37. 2013, Rio de Janeiro. Anais... ANPAD: Rio de Janeiro, 2013.

WOON, L. F.; AZIZAN, N. A.; SAMAD, M. F. A. A strategic framework for value enhancing enterprise risk management. Journal of Global Business and Economics, v. 2, n. 1, p. 23-48, 2011. 